\title{
TORSION-FREE PRE-IMAGES OF MODULES OF FINITE LENGTH
}

\author{
by L. O'CARROLL \\ (Received 17th December 1986)
}

\section{Introduction}

The purpose of this note is to provide a simplified proof of the following result due to Crawley-Boevey, Kropholler and Linnell.

Theorem ([1]). Let $A$ be a finitely generated commutative ring (with identity), and let $M$ be a finitely generated A-module which is torsion-free as a $\mathbb{Z}$-module. Given an $A$-submodule $U$ of $M$ such that $M / U$ is of finite length as an A-module, there is an $A$-submodule $V$ of $U$ such that $M / V$ is torsion-free as a $\mathbb{Z}$-module and such that $\mathbb{Q} \otimes{ }_{\mathrm{z}} M / V$ is a finite-dimensional $\mathbb{Q}$-vector space.

This result forms one step in a programme of results on zero-divisors in classes of group-rings, and the original proof involved induction on the number of generators of $M$ and a further induction involving associated primes. Here we use basic ideas in commutative algebra to circumvent these aspects of the original proof; specifically we use systems of parameters to engineer a situation in which one can apply a basic theorem from Zariski-Samuel [4] on the closure of a submodule in an ideal-adic topology. However we still employ one fundamental idea from the original, namely the use of Chevalley's theorem.

We would like to thank Peter Kropholler for bringing this result to our attention, and for giving a sketch of the original proof.

\section{The proof}

Without loss of generality $A=\mathbb{Z}\left[X_{1}, \ldots, X_{n}\right]$, where the $X_{i}$ are indeterminates. Since $M / U$ is of finite length, Ass $A / U=\left\{m_{1}, \ldots, m_{r}\right\} \subseteq \operatorname{MaxSpec} A$, and there exists a positive integer $s$ such that $\prod_{i} m_{i}^{s} M \subseteq U$. Suppose that we know the result to be true in the case where $\mid$ Ass $_{A} M / U \mid=1$, Ass $_{A} M / U=\{m\}$ say, where $m \in \operatorname{MaxSpec} A$, and $U=m^{s} M$.

By the Chinese Remainder Theorem

$$
M / \bigcap_{i}\left(m_{i}^{s} M\right) \cong \bigoplus_{i}\left(M / m_{i}^{s} M\right)
$$

and

$$
\bigcap_{i}\left(m_{i}^{s} M\right)=\left(\bigcap_{i} m_{i}^{s}\right) M=\left(\prod_{i} m_{i}^{s}\right) M \text {. }
$$


By assumption, for each $i$, there is an $A$-submodule $V_{i}$ of $m_{i}^{q} M$ such that $M / V_{i}$ is $\mathbb{Z}$-torsion-free and such that $\mathbb{Q} \otimes_{\mathrm{z}} M / V_{i}$ is a finite-dimensional $\mathbb{Q}$-vector-space. Set $V=\bigcap_{i} V_{i}$.

Then $V \subseteq U$ and $M / V \subset \bigoplus_{i} M / V_{i}$, and it is immediate that $M / V$ is $\mathbb{Z}$-torsion-free and that $\mathbb{Q} \otimes_{\mathrm{z}} M / V$ is a finite-dimensional $\mathbb{Q}$-vector space.

Hence it suffices to treat the case where Ass, $M / U=\{m\} \subseteq \operatorname{MaxSpec} A$ and $U=m^{8} M$.

For simplicity, set $B=A_{m}, C=\hat{A}_{m}$ (the $m$-adic completion of $A$ ), $N=M_{m}$ and $L=\hat{M}_{m}$ (the $m$-adic completion of $M$ ). We remark that $N \subseteq L$ as $L$ is also the $m_{m}$-adic completion of the $A_{m}$-module $N$. Since $A$ is a Hilbert ring, $m \cap \mathbb{Z}=(p)$ for some prime $p$ (and by Nullstellensatz, $M / U$ is in fact finite). Let $S=\mathbb{Z} \backslash 0$. Then $S^{-1} B=B_{p}, S^{-1} C=C_{p}$, $S^{-1} N=N_{p}$ and $S^{-1} L=L_{p}$.

Let Ass $_{S^{-1} C} S^{-1} L=\left\{Q_{1}, \ldots, Q_{t}\right\}$, and fix $i$ such that $1 \leqq i \leqq t$. Then $Q_{i}=S^{-1} Q_{i}^{\prime}$ for some $Q_{i}^{\prime} \in$ Ass $_{C} L$; setting $P_{i}=Q_{i}^{\prime} \cap B$, we have that $Q_{i}^{\prime} \in \operatorname{Ass}_{C} C / P_{i} C$ by [2, (9.B), Theorem 12(ii)]. Now $B$ is a local Cohen-Macaulay ring, so $B / P_{i}$ is unmixed in the sense of Nagata (see [3, p. 82 and (34.9)]). Thus $Q_{i}^{\prime}$ is minimal over $P_{i} C$ and

$$
\operatorname{co-rank}_{B} P_{i}=\operatorname{co-rank}_{C} Q_{i}^{\prime}=d
$$

(say), where $d$ depends on $i$. Since $S^{-1} C=C_{p}, p \notin Q_{i}^{\prime}$ so $p \notin P_{i}$.

Consider the embedding $B / P_{i} \subseteq C / Q_{i}^{\prime}$, and use "-" to denote corresponding images in $B / P_{i}$ (and $\left.C / Q_{i}^{\prime}\right)$. Now there is a set of $d$ elements $\left\{p_{1}, \ldots, p_{d}\right\}$ of $B$, with $p=p_{1}$, such that $\left\{\bar{p}_{1}, \ldots, \bar{p}_{d}\right\}$ forms a system of parameters for $B / P_{i}$ and $C / Q_{i}^{\prime}$. Let $I_{i}$ be a prime ideal in $C$ such that $I_{i} \supseteq Q_{i}^{\prime}$ and such that $I_{i} / Q_{i}^{\prime}$ is minimal over $\left\{\bar{p}_{2}, \ldots, \bar{p}_{d}\right\}$; set $J_{i}=I_{i} \cap B$. Then $S^{-1} I_{i}$ and $S^{-1} J_{i}$ are maximal ideals of $S^{-1} C$ and $S^{-1} B$ respectively.

Now let $i$ vary, and set $I=I_{1} \cap \cdots \cap I_{t}$ and $J=J_{1} \cap \cdots \cap J_{t}$, so that $J=I \cap B$. Then $S^{-1} I$ and $S^{-1} J$ are of co-rank 0 in $S^{-1} C$ and $S^{-1} B$ respectively. Moreover, $S^{-1} I$ is not co-prime to any $Q_{i}, 1 \leqq i \leqq t$. Consider the $S^{-1} I$-adic topology on $S^{-1} C$ and consider the zero-submodule of the $S^{-1} C$-module $S^{-1} L$. Since $S^{-1} I$ is not co-prime to any $Q_{i}$, $1 \leqq i \leqq t$, where the latter are the associated prime ideals of the zero-submodule, it follows from [4, p.261, Theorem 8] (cf. the first two lines of its proof) that

$$
\bigcap_{u \geqq 1} S^{-1} I^{u} L=\bigcap_{u \geqq 1}\left(S^{-1} I\right)^{u} S^{-1} L=0 .
$$

Now $S^{-1} L=L_{p} \supseteq L$, since $L$ is a torsion-free $\mathbb{Z}$-module by the flatness of the extension $A \rightarrow C$, so

$$
\bigcap_{u \geq 1} K_{u}=0
$$

where $K_{u}=\left(S^{-1} I^{u} L\right) \cap L$. (It is at this point that we use the $\mathbb{Z}$-torsion freeness of $M$.) By Chevalley's theorem [3, p. 105, Exer. 2], there exists a positive integer $v$ such that (in the usual notation)

$$
K_{v} \subseteq \hat{m}_{m}^{s} L
$$


Now contract to $N$, and pull back along the natural map $M \rightarrow M_{m}$, to get (by an easy proof)

$$
K_{v}^{\prime} \subseteq m^{s} M
$$

where $K_{v}^{\prime}$ is the pre-image in $M$ of $K_{v} \cap N$. (Recall that $N \subseteq L$.)

Clearly $K_{v}^{\prime}$ is the pre-image of $S^{-1} I^{v} L$ under the composite of the natural maps

$$
M \rightarrow N \subseteq L \subseteq S^{-1} L .
$$

Hence $M / K_{v}^{\prime} \cong S^{-1} L / S^{-1} I^{v} L$, so $M / K_{v}^{\prime}$ is $\mathbb{Z}$-torsion free. Moreover, $S^{-1}\left(M / K_{v}^{\prime}\right)$ is annihilated by $S^{-1}(J \cap A)^{\nu}$, an ideal of co-rank 0 in $S^{-1} A$. (Note that $S^{-1} A=\mathbb{Q}\left[X_{1}, \ldots, X_{n}\right]$ and that each $J_{i}$ is of rank $n$ in $B$.) This, together with (*), gives the result.

\section{REFERENCES}

1. W. W. Crawley-Boevey, P. H. Kropholler and P. A. Linnell, Torsion-free soluble groups, completions and the zero-divisor conjecture, J. Pure Appl. Algebra, to appear.

2. H. Matsumura, Commutative Algebra (2nd ed.) (Benjamin/Cummings, Reading, Massachusetts, 1980).

3. M. Nagata, Local Rings (Interscience Publishers, New York and London, 1962).

4. O. Zariski and P. SAmuel, Commutative Algebra Vol. II (Springer-Verlag, New York, 1960).

Department of Mathematics

UNIVERSITY OF EDINBURGH

James Clerk Maxwell Building

Mayfield Road

EDINBURGH EH9 3JZ 